\title{
A Case of Retinitis Pigmentosa Requiring Vitrectomy because of Repeated Vitreous Hemorrhage
}

\author{
Akira Watanabe Goichi Akiyama Hiroshi Tsuneoka \\ Department of Ophthalmology, The Jikei University School of Medicine, Tokyo, Japan
}

\section{Key Words}

Retinitis pigmentosa $\cdot$ Vitrectomy $\cdot$ Vitreous hemorrhage $\cdot$ Neovascularization

\begin{abstract}
A male patient with retinitis pigmentosa (RP) accompanied by repeated vitreous hemorrhage in both eyes underwent unilateral vitrectomy. Visual acuity recovered to 0.8 in the operated right eye, and no hemorrhage, complications or progression of RP were observed. Fluorescein angiography performed 2 months after surgery detected neovascularization at the optic disc in the operated right eye, but not in the nonoperated left eye, and no avascular areas were found in either eye. Vitrectomy may be effective for the treatment of RP accompanied by vitreous hemorrhage.
\end{abstract}

\section{Introduction}

Cataract, glaucoma, cystoid macular edema, and macular hole are complications of retinitis pigmentosa (RP). RP can also be accompanied by vitreous hemorrhage [1-4], albeit more rarely. Here, we report a case of $\mathrm{RP}$ with repeated vitreous hemorrhage for which we performed vitrectomy.

\section{Case Presentation}

A 27-year-old male, who had been first diagnosed with RP at the age of 8 years, was being routinely followed. He presented with concentric visual field constriction to $10^{\circ}$ and flattening of the waves on electroretinograms. His father had a history of RP. The patient suffered from vitreous hemorrhage at the age of 19, and although the bleeding spontaneously resolved in around 1 month with an improvement in vision, he was routinely followed up. Since the first hemorrhage, vitreous hemorrhage has recurred numerous times followed by spontaneous recovery in around 1 month.

In May 2010, vitreous hemorrhage recurred in the patient's right eye and the bleeding was extensive. Because no improvement was seen after 1 month, the patient was referred to the Department of Ophthalmology at the Jikei University Hospital, Tokyo, Japan. 
The initial medical examination at our hospital revealed that visual acuity of the right eye was hand movements and for the left eye it was $1.2 \times-2.75 \mathrm{D}$ cyl $-3.00 \mathrm{DA} \times 180^{\circ}$. Intraocular pressure was $14 \mathrm{~mm}$ $\mathrm{Hg}$ in the right eye and $16 \mathrm{~mm} \mathrm{Hg}$ in the left eye. Because vitreous hemorrhage in the right eye showed no improvement at the 1-month follow-up observation, we performed a 23-gauge vitrectomy on July 27 , 2010. During surgery, intraocular endoillumination as well as microscope illumination were set as low as possible, and the infusion pressure was set at $25 \mathrm{~mm} \mathrm{Hg}$ (fig. 1). Dense hemorrhage and adhesion of the optic disc and the vitreous body were observed. Posterior vitreous detachment was present in other areas. Bone spicule-like pigmentation and blood vessel constriction were remarkable in the ocular fundus from the vascular arcades to the equator, with no proliferative changes. The lens was preserved, and the vitreous body around the port was removed by compressing the sclera. Operation time was 40 $\mathrm{min}$. After surgery, visual acuity of the right eye recovered to $0.8 \times-3.25 \mathrm{D}$ cyl $-3.25 \mathrm{DA} \times 10^{\circ}$, and no postoperative complications were observed.

Fig. 2 shows the results of fluorescein angiography (FA) performed 2 months after surgery. In both eyes, we observed atrophy of the pigment epithelium except in the macula, constriction of retinal vessels, appearance of pigmentation, and minor vascular leakage. In the right eye, we observed atrophy of the choroidal capillary plate seen below the optic disc. In the late phase, neovascularization was observed over the optic disc, which was thought to be the cause of the previous vitreous hemorrhage. In the left eye, there were no findings of neovascularization or anything that might cause bleeding. No avascular areas were present in either eye.

Kinetic visual field testing performed 3 months after surgery revealed no postoperative changes in the visual field (fig. 3 ).

In the right eye, neither recurrence of vitreous hemorrhage nor change in the neovascular vessels over the optic disc has been observed for 10 months after surgery. Although the left eye has shown repeated vitreous hemorrhage, the blood usually absorbs without treatment in around 1 month; therefore, the left eye is currently under observation.

\section{Discussion}

Various complications of RP have been reported, and cases of vitreous hemorrhage accompanying RP are occasionally encountered. Neovascularization, retinal vascular abnormalities [1-4], and Coats'-like exudative retinal detachment [5-7] are the mechanisms reportedly associated with vitreous hemorrhage in RP.

In the present case, FA detected neovasculature over the optic disc, but no avascular areas, in the right eye after surgery. In chronic inflammatory diseases, neovascularization is occasionally seen in absence of non-perfused areas [8]. In our RP case, the patient had suffered persistent chronic inflammation, and inflammatory cells were at times detected in the vitreous body, both of which might have led to the formation of the neovasculature.

Similar to the right eye, the left eye has repeatedly shown vitreous hemorrhage. However, FA did not detect any non-perfused areas or neovascularization, and thus the cause of the hemorrhage is currently unknown. A lesion may be present in the extreme periphery of the retina, the area not covered by FA, or it is possible that a pathological condition is present which cannot be detected by FA. Nao-i et al. [4] reported FA findings of neovascular and surrounding avascular formation in a case of vitreous hemorrhage presenting 2 years after no initial findings of neovascularization or avascular areas had been seen on FA. For this reason, we will continue with the careful observation of the patient and provide treatment if the formation of neovascular or avascular areas appears.

Potential treatments for vitreous hemorrhage accompanying RP include photocoagulation, intravitreal injection of the anti-vascular endothelial growth factor (VEGF), and vitrectomy combined with regular follow-up observations. Anti-VEGF 
treatment is considered useful for regression of the neovasculature when hemorrhage is caused by neovascularization. However, some questions remain as to whether anti-VEGF treatment presents no problem for RP.

In the present case of vitreous hemorrhage, surgery was performed because the patient suffered frequent recurrence, and the last episode of bleeding was not resolved within 2 months. There are, however, cases where vitreous hemorrhage improves after long-term follow-up observation with no recurrence of bleeding $[1,2,4-7]$. Therefore, at the time of first bleeding, it is necessary to consider the potential adverse effects of vitrectomy on the progression of RP and decide the length of the follow-up observation.

There are reports of vitrectomy performed on patients with macular disease, such as macular hole and macular edema $[9,10]$, accompanying $\mathrm{RP}$, which led to a reduction in the amplitude of the multifocal electroretinogram and decreased visual acuity after surgery. On the other hand, Hayashi et al. [3] performed vitrectomy in a case of vitreous hemorrhage without any postoperative complications or RP progression. Similarly, in the present case, there has been no noticeable progression of RP during the 10 months of follow-up after surgery. Kinetic visual field testing performed 3 months after surgery also revealed no postoperative changes in the visual field (fig. 3). However, the indication for vitrectomy in RP needs to be determined carefully, provided that there are no other complications besides vitreous hemorrhage, as in the present case, and that surgery is performed with surgical stress such as damage from endoillumination and infusion pressure minimized, it appears that vitrectomy can be an effective treatment for vitreous hemorrhage in RP.

\section{Disclosure Statement}

None of the authors has any financial interests concerning procedures or drugs cited in this case report. 


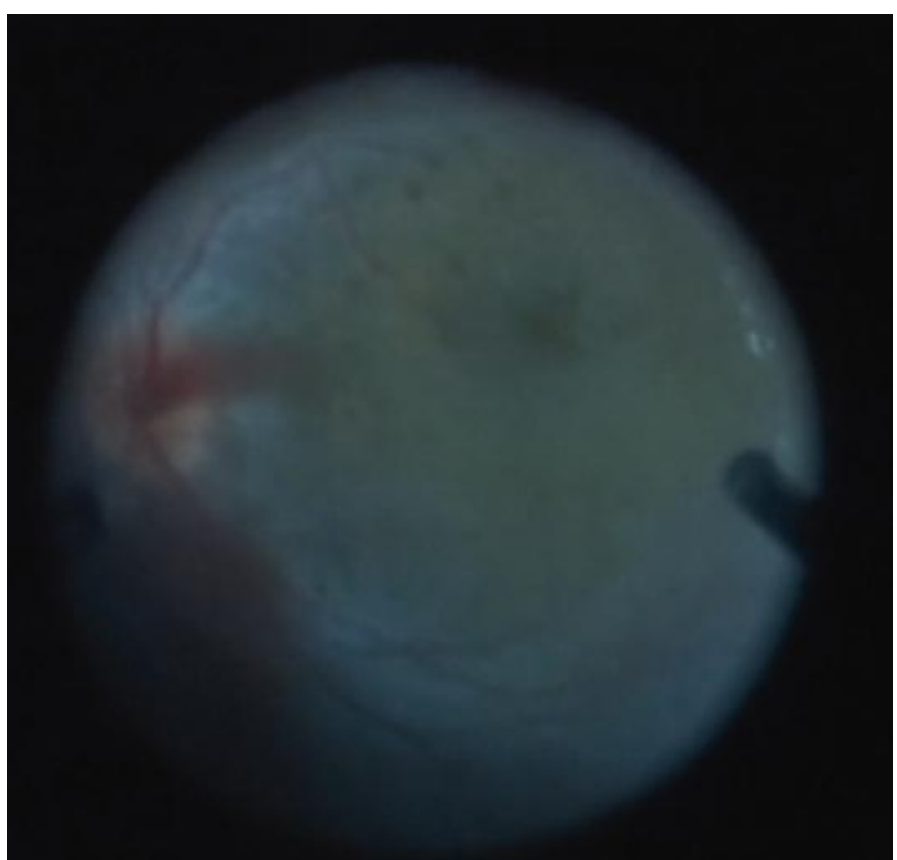

Fig. 1. During vitrectomy, intraocular endoillumination was set as low as possible. 


\section{Case Reports in Ophthalmology}

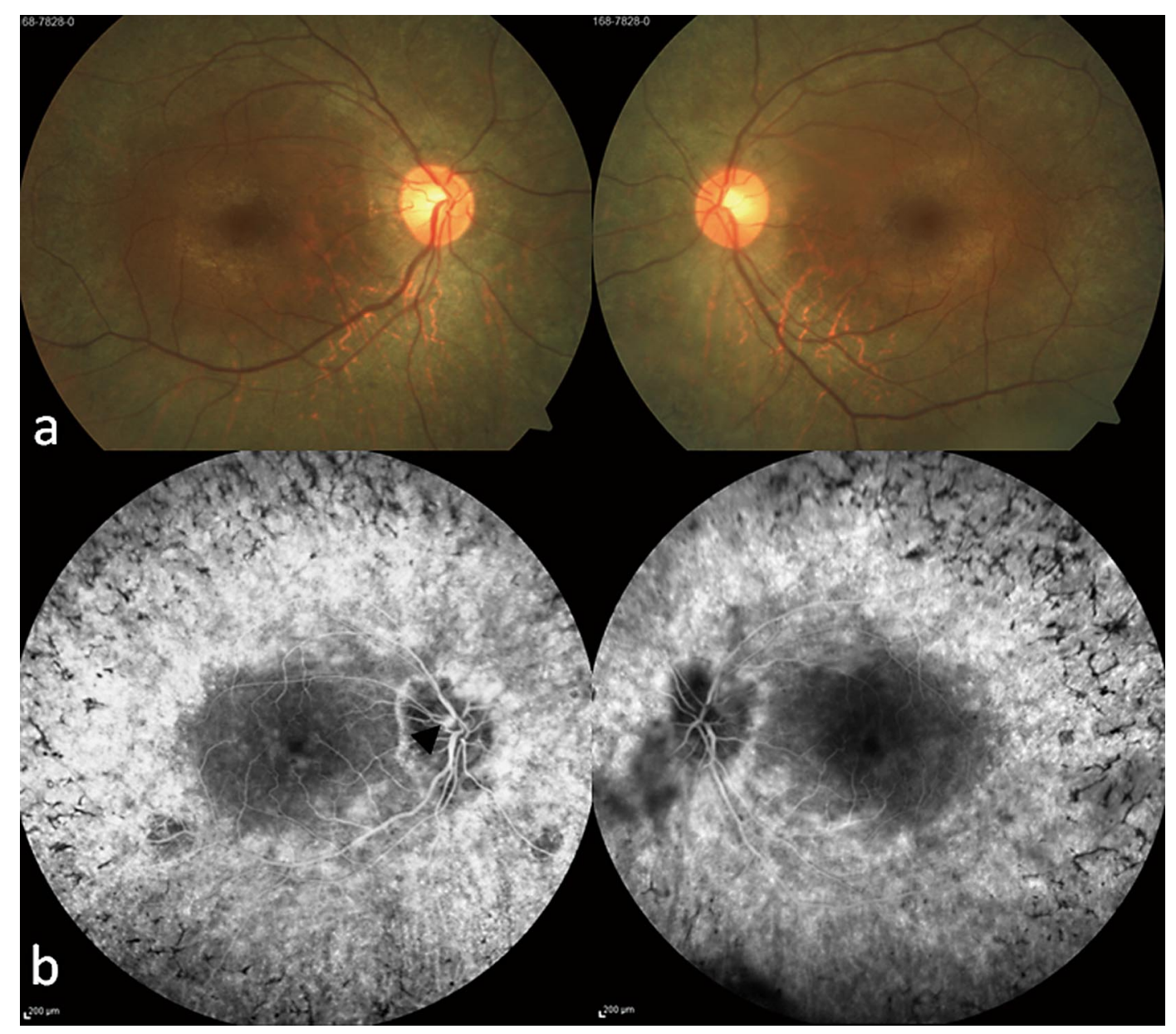

Fig. 2. a Fundus photograph. b FA performed 2 months after vitrectomy. In the right eye, neovascularization was observed over the optic disc (black arrowhead). 

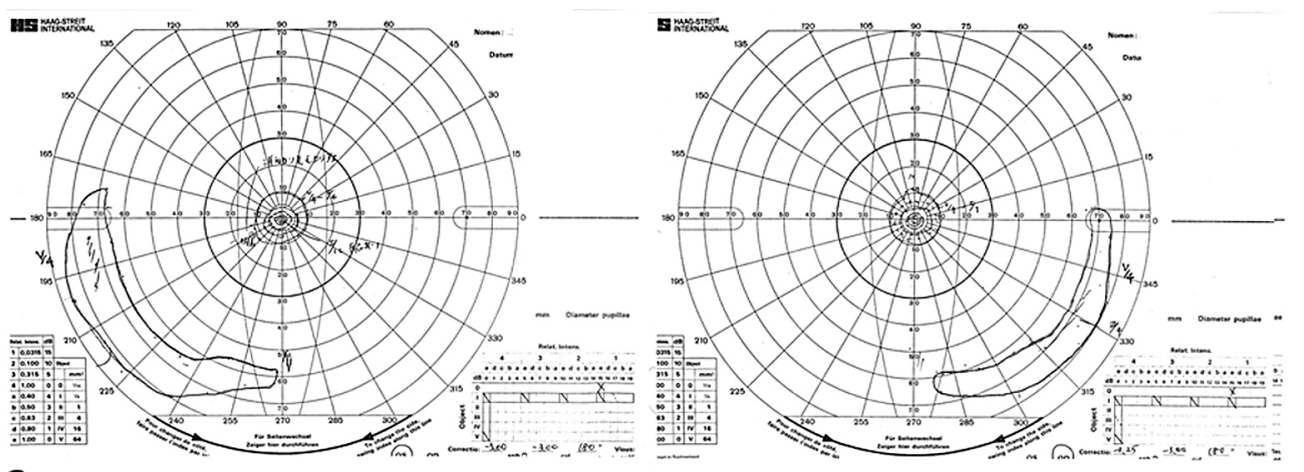

a
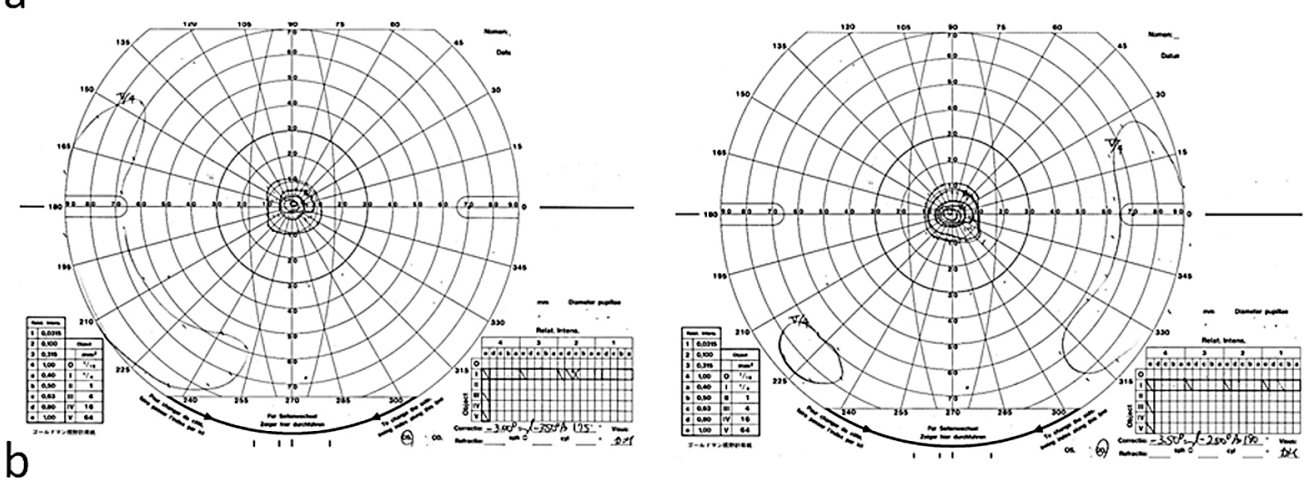

Fig. 3. Kinetic visual field testing performed 3 months after surgery revealed no postoperative changes in the visual field. a Before vitrectomy. $\mathbf{b}$ Three months after vitrectomy.

\section{References}

1 Uliss AE, Gregor ZJ, Bird AC: Retinitis pigmentosa and retinal neovascularization. Ophthalmology 1986;93:1599-1603.

2 Bressler NM, Gragoudas ES: Neovascularization of the optic disk associated with atypical retinitis pigmentosa. Am J Ophthalmol 1985;100:431-433.

3 Hayashi S, Hayashi L, Ise T, Suzuki K, Chikuda M: A case of retinitis pigmentosa with disc neovascularization treated by vitrectomy [in Japanese]. Jpn J Clin Ophthalmol 2001;55:991-995.

-4 Nao-i N, Fukiyama J, Sawada A: Retinitis pigmentosa with recurrent vitreous hemorrhage. Acta Ophthalmol Scand 1996;74:509-512.

-5 Fogle JA, Welch RB, Green WR: Retinitis pigmentosa and exudative vasculopathy. Arch Ophthalmol 1978;96:696-702.

6 Medlock RD, Shields JA, Shields CL, Yarian DL, Beyrer CR: Retinal hemangioma-like lesions in eyes with retinitis pigmentosa. Retina 1990;10:274-277.

7 Demirci FY, Rigatti BW, Mah TS, Gorin MB: A novel RPGR exon ORF15 mutation in a family with X-linked retinitis pigmentosa and Coats'-like exudative vasculopathy. Am J Ophthalmol 2006;141:208-210.

$>8$ Henkind P: Ocular neovascularization. Am J Ophthalmol 1978;85:287-301.

-9 Hagiwara A, Yamamoto S, Ogata K, Sugawara T, Hiramatsu A, Shibata M, Mitamura Y: Macular abnormalities in patients with retinitis pigmentosa: prevalence on OCT examination and outcomes of vitreoretinal surgery. Acta Ophthalmol 2011;89:122-125.

10 Jin ZB, Gan DK, Xu GZ, Nao-i N: Macular hole formation in patients with retinitis pigmentosa and prognosis of pars plana vitrectomy. Retina 2008;28:610-614. 